\title{
Knudsen Corrections for Flow Birefringence in Gases
}

\author{
H. Vestner \\ Institut für Theoretische Physik der Universität Erlangen-Nürnberg, Erlangen
}

(Z. Naturforsch. 29 a, 365-372 [1974] ; received 28 August 1973)

\begin{abstract}
Differential equations and boundary conditions for the flow velocity and the tensor polarization are solved for a cylindrical Couette-flow apparatus in a magnetic field. The ensuing Knudsen corrections for flow birefringence in gases consist of two contributions: (i) the velocity gradient is reduced by slip of the gas at the walls, (ii) the tensor polarization itself is influenced by gas-wall collisions. As a result, flow birefringence decreases for low pressures. For the gases $\mathrm{HD}, \mathrm{N}_{2}$ and $\mathrm{CO}$ the relevant surface parameters are already known from Knudsen corrections for the Senftleben-Beenakker effect of viscosity. Thus the explicit pressure dependence of flow birefringence can be predicted for these gases (for zero magnetic field) and for the apparatus used by Baas.
\end{abstract}

In 1971 Baas ${ }^{1}$ reported results of the first flow birefringence measurements in gases, in particular in $\mathrm{CO}_{2}$. For the difference in the index of refraction $\delta v$ he found ${ }^{1} 10^{-13}$ as order of magnitude. Over a wide pressure range $\delta v$ was independent of pressure in accordance with the kinetic theory developed by Hess ${ }^{2-4}$. But for pressures lower than 0.1 torr he observed $^{1}$ a decrease of $\delta v$ due to Knudsen effects. The subject of this paper is the calculation of these Knudsen corrections for flow birefringence. To this end the kinetic theory of Hess ${ }^{2-4}$ and of St. Pierre, Köhler and Hess ${ }^{5}$, who incorporated a magnetic field, is extended to include wall effects which are typical for a rarefied gas. The method applied here is the same which previously had been used for a calculation of Knudsen corrections for the Senftleben-Beenakker effect of viscosity ${ }^{6,7}$. From there values for the relevant surface parameters have been obtained ${ }^{6}$.

Pure gases of rotating diatomic molecules have been considered in the theories of Hess ${ }^{2-4}$ and of St. Pierre et al. ${ }^{5}$. Recently, flow birefringence in gaseous mixtures has been studied by Köhler and Halbritter ${ }^{8}$.

A kinetic theory of heat-flow birefringence in rarefied gases has been worked out by Hess ${ }^{9}$.

The basic ideas for a theory of birefringence phenomena in polyatomic gases are summarized as follows. By collisions the rotational angular momenta of the molecules can be oriented, hence polarizations will occur in a non-equilibrium situation. The existence of these polarizations is indicated indirectly by the influence of a magnetic field on flow

Reprint requests to Dr. H. Vestner, Institut für Theoretische Physik der Universität Erlangen-Nürnberg, D-8520 Erlangen, Glückstraße 6. and heat conduction in polyatomic gases, often referred to as Senftleben-Beenakker effect ${ }^{10}$. In this connection the most important types of alignment are ${ }^{10}$ the tensor polarization and the Kagan polarization (i.e. the flux of tensor polarization) for a streaming and a heat conducting gas, respectively. Starting point for a theoretical treatment of polarization phenomena in gases is the quantum mechanical kinetic equation due to Waldmann and Snider ${ }^{11}$. By application of the moment method ${ }^{12}$ to the linearized Waldmann-Snider equation differential equations for macroscopic variables are derived. In the hydrodynamical regime the tensor polarization is found to be proportional to the velocity gradient. Now, for a gas of rotating diatomic molecules the anisotropic part $\overline{\boldsymbol{\epsilon}}$ of the dielectric tensor is proportional to the tensor polarization ${ }^{2-4}$, and hence it is also proportional to the velocity gradient. Thus, flow birefringence will occur. The relevant coefficient $\beta$ in the relation between $\overline{\boldsymbol{\epsilon}}$ and the velocity gradient is expressed by collision integrals of the linearized Waldmann-Snider collision term. In this way relations between flow birefringence and the Senftleben-Beenakker effect of viscosity are established ${ }^{2-4}$.

In all these theories ${ }^{2-5,8}$ for the hydrodynamical regime the coefficient $\beta$ of flow birefringence and the difference $\delta v$ in the index of refraction are independent of pressure. For a calculation of Knudsen corrections of $\beta$ and $\delta v$ nonlocal effects within the gas have to be taken into account, i. e. terms proportional to the mean free path of a molecule can no longer be neglected. Especially, in a streaming gas the Kagan polarization has to be considered too besides the tensor polarization. Consequently, a second order spatial differential equation for the 
tensor polarization is obtained ${ }^{6,7}$ instead of an explicit linear relation ${ }^{2-5}$. Then, also a boundary condition for the tensor polarization is needed. Phenomenological boundary conditions for macroscopic variables (e.g. the velocity and the tensor polarization) are derived by Waldmann's thermodynamic method ${ }^{13}$ from the entropy production at the interface between the gas and a solid body ${ }^{6,7}$.

This paper is divided into three major sections. In Section 1 constitutive laws for the friction pressure tensor and the Kagan polarization are stated, then differential equations and boundary conditions for the velocity and the tensor polarization are given. Section 2 deals with the calculation of the velocity and the tensor polarization for a Couette-flow apparatus in a magnetic field. The Knudsen corrections for flow birefringence are discussed in Section 3 . Especially, for zero magnetic field the difference $\delta v$ in the index of refraction is considered in detail. The low pressure dependence of $\delta v$ is given explicitly for the experimental set up used by Baas ${ }^{1}$ and for the gases HD, $\mathrm{N}_{2}$ and CO. This is possible since the relevant surface parameters characterizing the influence of gas-wall collisions on the tensor polarization are already known ${ }^{6}$ from Knudsen corrections for the Senftleben-Beenakker effect of viscosity.

\section{Basic Equations}

\section{(a) Definition of the moments}

For a gas of rotating linear molecules the anisotropic part $\overline{\boldsymbol{\epsilon}}$ of the dielectric tensor is related to the tensor polarization $\mathbf{a}$ by ${ }^{2-4}$

$$
\overline{\boldsymbol{\epsilon}}=\varepsilon^{\prime} \mathbf{a} .
$$

The tensor polarization is a symmetric traceless tensor of rank 2 built up from the rotational angular momentum $\hbar \boldsymbol{J}$ of a molecule ${ }^{2-4}$ :

$$
\begin{aligned}
\mathbf{a} & =\langle\boldsymbol{\Phi}\rangle, \\
\mathbf{\Phi} & =\sqrt{\frac{15}{2}}\left(\left\langle J^{2}\left(J^{2}-\frac{3}{4}\right)^{-1}\right\rangle_{0}\right)^{-1 / 2}\left(J^{2}-\frac{3}{4}\right)^{-1} \overline{\boldsymbol{J} \mathbf{J}} .
\end{aligned}
$$

Here, the bracket $\langle\ldots\rangle$ refers to a nonequilibrium average, $\langle\ldots\rangle_{0}$ is evaluated with the equilibrium distribution. By the symbol $\ldots$ the symmetric traceless part of a tensor is denoted. The coefficient $\varepsilon^{\prime}$ depends on the density $n$ and on the difference between the polarizabilities parallel $(\alpha \|)$ and perpendicular $\left(\alpha_{\perp}\right)$ to the molecular axis:

$$
\varepsilon^{\prime}=-2 \pi n\left(\alpha_{\|}-\alpha_{\perp}\right) \sqrt{\frac{2}{15}}\left(\left\langle J^{2}\left(J^{2}-\frac{3}{4}\right)^{-1}\right\rangle_{0}\right)^{1 / 2} .
$$

For a calculation of Knudsen corrections the Kagan polarization (i. e. the tensor polarization flux)

$$
b_{\lambda, \mu \nu}=\left\langle c_{\lambda} \Phi_{\mu \nu}\right\rangle \sqrt{M / k} T_{0}
$$

has to be taken into account too. Here, $\boldsymbol{c}$ denotes the molecular velocity and $M$ the mass of a molecule, $k$ is Boltzmann's constant and $T_{0}$ the constant temperature of the gas.

\section{(b) Constitutive laws and differential equations}

The nonequilibrium state of the gas is described by the flow velocity, the friction pressure tensor $\overline{\mathbf{p}}$, the tensor polarization $\mathbf{a}$, and the tensor polarization flux b. By application of the moment method ${ }^{12}$ to the linearized Waldmann-Snider equation transport relaxation equations for these quantities are derived. In a steady state, they supply us with ${ }^{6,7}$ constitutive laws for the friction pressure tensor

$$
\overline{\mathbf{p}}=-2 \eta_{\text {iso }} \overline{\nabla \boldsymbol{v}}-p_{0} \sqrt{2} \omega_{\eta \mathrm{T}} / \omega_{\eta} \mathbf{a},
$$

and the Kagan polarization

$b_{\lambda, \mu \nu}=-l_{\mathrm{b}} \frac{\partial}{\partial x_{\lambda}} \sum_{m=-2}^{+2} \frac{1}{1+i m \varphi_{\mathrm{b}}} \mathcal{F}_{\mu \nu, \mu^{\prime} \nu^{\prime}}^{(m)} a_{\mu^{\prime} v^{\prime}}$.

Furthermore, differential equations are obtained for the flow velocity $\boldsymbol{v}\left(p=n k T_{0}\right.$ is the pressure)

$$
\nabla \cdot \boldsymbol{v}=0, \frac{1}{\eta_{\mathrm{iso}}} \nabla p-\nabla \cdot \nabla \boldsymbol{v}=\sqrt{2} \omega_{\eta \mathrm{T}} \nabla \cdot \mathbf{a},
$$

and for the tensor polarization $\mathbf{a}$

$$
\begin{aligned}
& \left(1-L_{m}^{2} \nabla \cdot \nabla\right) \mathcal{P}^{\mathcal{P}(m)}: \mathbf{a} \\
& =\frac{\sqrt{2} \omega_{\eta \mathrm{T}}}{\omega_{\eta} \omega_{\mathrm{T}}} \frac{1}{1+i m \varphi_{\mathrm{T}}} \mathrm{p}^{(m)}: \bar{\nabla} \boldsymbol{v}, m=0, \pm 1, \pm 2 \text {. } \\
& \text { Notice }^{3,14} \quad \mathbf{a}=\sum_{m=-2}^{+2} \mathcal{P}(m): \mathbf{a} .
\end{aligned}
$$

The relaxation constants $\omega_{\eta}, \omega_{\mathrm{T}}, \omega_{\eta \mathrm{T}}$ and $\omega_{\mathrm{b}}$ are collision integrals of the linearized Waldmann-Snider collision term ${ }^{3,4}, 9$. Experimentally they can be determined from the shear viscosity $\eta\left(\omega_{\eta}\right)$,

$$
\eta=\eta_{\text {iso }}\left(1+\frac{\omega_{\eta \mathrm{T}}^{2}}{\omega_{\eta} \omega_{\mathrm{T}}}\right), \eta_{\text {iso }}=\frac{p_{0}}{\omega_{\eta}}, \frac{\eta-\eta_{\text {iso }}}{\eta_{\text {iso }}} \ll 1,
$$

and from the Senftleben-Beenakker effects of viscosity $\left(\omega_{\mathrm{T}},\left|\omega_{\eta \mathrm{T}}\right|\right)$ and of thermal conductivity $\left(\omega_{\mathrm{b}}\right)$.

A magnetic field $\boldsymbol{H}=H \boldsymbol{h}(\boldsymbol{h} \cdot \boldsymbol{h}=1)$ enters via the precession angles $\varphi_{\mathrm{T}}, \varphi_{\mathrm{b}}(\gamma$ is the rotational gyromagnetic ratio)

$$
\varphi_{\mathrm{T}}=\omega_{\mathrm{H}} / \omega_{\mathrm{T}}, \varphi_{\mathrm{b}}=\omega_{\mathrm{H}} / \omega_{\mathrm{b}}, \omega_{\mathrm{H}}=\gamma H,
$$


and via the fourth rank projection tensors ${ }^{3,14} \mathcal{P}(m)$, $m=0, \pm 1, \pm 2$, which contain the field direction $\boldsymbol{h}$.

In Eqs. (1.6) and (1.8) the mean free paths

$l_{b}=\sqrt{k T_{0}} / M / \omega_{\mathrm{b}}, L_{\mathrm{m}}=\sqrt{k T_{0} / M \omega_{\mathrm{T}} \omega_{\mathrm{b}}}$

$\cdot\left[\left(1+i m \varphi_{\mathrm{T}}\right)\left(1+i m \varphi_{\mathrm{b}}\right)\right]^{-1 / 2}, m=0, \pm 1, \pm 2$,

indicate the presence of Knudsen effects. These contributions vanish for $l_{\mathrm{b}}=0, L_{\mathrm{m}}=0$, then $\underline{\mathbf{b}}=0$ applies and Eq. (1.8) reduces to an explicit ansatz for the tensor polarization. This is appropriate for the hydrodynamical regime which has been studied up to now ${ }^{2-5,8}$. But here, the behaviour of a rarefied gas shall be investigated, i. e. $l_{\mathrm{b}} \neq 0, L_{\mathrm{m}} \neq 0$ apply. Thus the tensor polarization flux $\underline{\mathbf{b}}$ has to be retained, and the differential Eq. (1.8) for $\mathbf{a}$ has to be solved. This can only be done if boundary conditions are available.

\section{(c) Boundary conditions}

Phenomenological boundary conditions for $\boldsymbol{v}$ and a are derived ${ }^{6,7}$ by Waldmann's thermodynamic method ${ }^{13}$ from the entropy production at the interface between the gas and a solid body. Here, the same simplified set is given which has been used for the calculation of Knudsen corrections for the Senftleben-Beenakker effect of viscosity ${ }^{6}$ :

$$
\begin{gathered}
\left(\boldsymbol{v}-\boldsymbol{v}_{\mathrm{solid}}\right)^{\tan }=C_{\mathrm{m}} \frac{l}{\eta_{\mathrm{iso}}} \boldsymbol{k}^{\mathrm{tan}}+ \\
\cdot \sqrt{\frac{k T_{0}}{M}} C_{\mathrm{ma}}(\boldsymbol{n} \cdot \underline{\mathbf{b}} \cdot \boldsymbol{n})^{\tan }, \\
\mathbf{a}=C_{\mathrm{am}} p_{0}^{-1} \overline{\boldsymbol{k}^{\tan } \boldsymbol{n}}+C_{\mathrm{a}} \boldsymbol{n} \cdot \underline{\mathbf{b}} .
\end{gathered}
$$

The velocity of the solid is denoted by $\boldsymbol{v}_{\text {solid }}$, $\boldsymbol{k}^{\tan }=(\overline{\mathbf{p}} \cdot \boldsymbol{n})^{\tan }$ is the tangential force the gas exerts per unit area of the solid, $\boldsymbol{n}$ is the outer unit normal of the gas, and

$$
l=\frac{4}{5} \sqrt{\frac{8 k T_{0}}{\pi M}} \omega_{\eta}^{-1}
$$

is the viscosity mean free path.

The dimensionless phenomenological parameters $C_{\mathrm{m}}, C_{\mathrm{a}}, C_{\mathrm{am}}, C_{\mathrm{ma}}$ characterize the gas-wall collisions. The mechanical slip coefficient $C_{\mathrm{m}}$ and the parameter $C_{\mathrm{a}}$ describe the accommodation of linear momentum and of tensor polarization, respectively. Due to the Onsager anti-symmetry relation $C_{\mathrm{am}}=$ $-C_{\mathrm{ma}}$ the coefficient of thermomagnetic slip, $C_{\mathrm{ma}}$, is linked to $C_{\mathrm{am}}$ which describes a production mecha- nism for the tensor polarization by gas-wall collisions. All these parameters have been determined ${ }^{6}$ for the gases $\mathrm{HD}, \mathrm{N}_{2}$ and $\mathrm{CO}$ from the Knudsen corrections observed for the Senftleben-Beenakker effect of viscosity ${ }^{10}$.

In the next section the flow velocity and the tensor polarization shall be calculated for a Couette-flow arrangement. Then in Sect. 3 Knudsen corrections for flow birefringence shall be discussed. All measurements of flow birefringence have been done ${ }^{1}$ without a magnetic field, but, since it is no difficulty, all calculations shall be performed with the inclusion of a field.

\section{Calculation of Velocity and Tensor Polarization}

According to Eq. (1.8) the tensor polarization is essentially determined by the velocity gradient, and in turn, the velocity is influenced by the presence of the tensor polarization, see Equation (1.7). Here, the tensor polarization shall be calculated in the most simple approximation from the "isotropic" flow field $\boldsymbol{v}_{\text {iso }}$, which is obtained from Eqs. (1.5), (1.7) and (1.12) by neglect of the polarizations $\mathbf{a}$ and $\underline{\mathbf{b}}$.

\section{(a) Isotropic flow velocity}

The experimental set up used by Baas ${ }^{1}$ is a cylindrical Couette-flow apparatus: the inner cylinder (of radius $R_{\mathrm{i}}$ ) is rotated with angular velocity $\Omega_{\mathrm{i}}$, the outer cylinder (with radius $R_{\mathrm{a}}$ ) is at rest. The unit vectors $\boldsymbol{e}$ and $\boldsymbol{u}$ are parallel and perpendicular to the cylinder axis respectively, hence $\boldsymbol{e} \times \boldsymbol{u}$ points in the azimuthal direction. A light beam is sent through the gas parallel to $\boldsymbol{e}$ at a distance $r\left(R_{\mathrm{i}}<r<R_{\mathrm{a}}\right)$ from the cylinder axis.

In a linear approximation the pressure is constant, $p=p_{0}$. Since the tensor polarization is neglected the differential equation (1.7) for the isotropic flow velocity

$$
\boldsymbol{v}_{\text {iso }}=V(r) \boldsymbol{e} \times \boldsymbol{u}
$$

reduces to

$\nabla \cdot \nabla \boldsymbol{v}_{\text {iso }}=\boldsymbol{e} \times \boldsymbol{u}\left(\frac{\mathrm{d}^{2} V}{\mathrm{~d} r^{2}}+\frac{1}{r} \frac{\mathrm{d} V}{\mathrm{~d} r}-\frac{V}{r^{2}}\right)=0$.

The solution has the form

$$
V(r)=c_{0} / r-c_{1} r
$$

hence we have

$$
\bar{\nabla}_{\text {iso }}=-\left(2 c_{0} / r^{2}\right) \widetilde{\boldsymbol{u} \boldsymbol{e} \times \boldsymbol{u}} .
$$


The constants $c_{0}$ and $c_{1}$ are determined by insertion of Eqs. (2.1), (2.2) into the boundary condition

$$
\left(\boldsymbol{v}_{\text {iso }}-\boldsymbol{v}_{\text {solid }}\right)^{\tan }=C_{\mathrm{m}}\left(l / \eta_{\text {iso }}\right) \boldsymbol{k}_{\text {iso }}^{\tan },
$$

which follows from (1.12) with $\underline{\mathbf{b}}=0$. In the isotropic approximation $\overline{\mathbf{p}}_{\text {iso }}=-2 \eta_{\text {iso }} \overline{\nabla \boldsymbol{v}}_{\text {iso }}$ applies, and $\boldsymbol{k}_{\text {iso }}^{\tan }=\left(\overline{\mathbf{p}}_{\text {iso }} \cdot \boldsymbol{n}\right)^{\tan }$ is calculated from (2.3) with $\boldsymbol{n}=\boldsymbol{u}$ for $r=R_{\mathrm{a}}, \boldsymbol{n}=-\boldsymbol{u}$ for $r=R_{\mathrm{i}}$. By the use of $\boldsymbol{v}_{\text {solid }}=\Omega_{\mathrm{i}} R_{\mathrm{i}} \boldsymbol{e} \times \boldsymbol{u}$ for $r=R_{\mathrm{i}}$ and $\boldsymbol{v}_{\text {solid }}=0$ for $r=R_{\mathrm{a}}$ the following result is obtained:

$$
\begin{aligned}
c_{0}= & \frac{\Omega_{\mathrm{i}}}{R_{\mathrm{i}}^{-2}-R_{\mathrm{a}}^{-2}} \frac{1}{1+2(l / d) C_{\mathrm{m}}}, \\
& \cdot \quad c_{1}=c_{0} R_{\mathrm{a}}^{-2}\left(1-2\left(l / R_{\mathrm{a}}\right) C_{\mathrm{m}}\right) .
\end{aligned}
$$

The length

$$
d=\left(R_{\mathrm{a}}{ }^{2}-R_{\mathrm{i}}{ }^{2}\right) R_{\mathrm{a}} R_{\mathrm{i}} /\left(R_{\mathrm{a}}{ }^{3}+R_{\mathrm{i}}{ }^{3}\right)
$$

is always smaller than the gap between the two cylinders, i. e. $0<d<R_{\mathrm{a}}-R_{\mathrm{i}}$.

In the high pressure limit $\left(p_{0} \rightarrow \infty, l=0\right)$ the velocity gradient is given by

$$
\left(\bar{\nabla}_{\text {iso }}\right)_{\infty}=-\frac{2 \Omega_{\mathrm{i}}}{r^{2}\left(R_{\mathrm{i}}^{-2}-R_{\mathrm{a}}{ }^{-2}\right)} \overline{\boldsymbol{u} \boldsymbol{e} \times \boldsymbol{u}} .
$$

For finite mean free path it is reduced due to mechanical slip

$$
\overline{\nabla \boldsymbol{v}}_{\mathrm{iso}}=\left(\bar{\nabla}_{\mathrm{iso}}\right)_{\infty}\left(1+2(l / d) C_{\mathrm{m}}\right)^{-1} .
$$

\section{(b) Tensor polarization}

Now, $\overline{\nabla \boldsymbol{v}}$ occurring on the right hand side of Eq. (1.8) is replaced by $\bar{\nabla}_{\text {iso }}$, cf. Equation (2.3). Then the following differential equations for the tensor polarization are obtained:

$$
\begin{gathered}
\left.\left(1-L_{\mathrm{m}}{ }^{2} \nabla \cdot \nabla\right)\right)^{\mathcal{P}(m)}: \mathbf{a}=-2 c_{0} r^{-2} \frac{\sqrt{2} \omega_{\eta \mathrm{T}}}{\omega_{\eta} \omega_{\mathrm{T}}} \\
\cdot\left(1+i m \varphi_{\mathrm{T}}\right)^{-1} \mathcal{P}^{(m)}: \overline{\boldsymbol{u} \boldsymbol{e} \times \boldsymbol{u}} .
\end{gathered}
$$

The ansatz

$\mathcal{P}^{(m)}: \mathbf{a}=-2 c_{0} \frac{\sqrt{2} \omega_{\eta \mathrm{T}}}{\omega_{\eta} \omega_{\mathrm{T}}} \tilde{A}_{\mathrm{m}}(r) \mathcal{P}^{(m)}: \overline{\boldsymbol{u} \boldsymbol{e} \times \boldsymbol{u}}$

yields

$$
\begin{array}{r}
\tilde{A}_{\mathrm{m}}-L_{\mathrm{m}}{ }^{2}\left(\frac{\mathrm{d}^{2} \tilde{A}_{\mathrm{m}}}{\mathrm{d} r^{2}}+\frac{1}{r} \frac{\mathrm{d} \tilde{A}_{\mathrm{m}}}{\mathrm{d} r}-\frac{4}{r^{2}} \tilde{A}_{\mathrm{m}}\right) \\
=r^{-2}\left(1+i m \varphi_{\mathrm{T}}\right)^{-1} .
\end{array}
$$

A special solution of this equation is given by $r^{-2}\left(1+i m \varphi_{\mathrm{T}}\right)^{-1}$, thus we can write

$$
\tilde{A_{\mathrm{m}}}(r)=r^{-2}\left(1+i m \varphi_{\mathrm{T}}\right)^{-1}+A_{\mathrm{m}}(r),
$$

and $A_{\mathrm{m}}(r)$ obeys the homogeneous differential equation

$$
\begin{aligned}
\frac{\mathrm{d}^{2} A_{\mathrm{m}}}{\mathrm{d} \varrho_{\mathrm{m}}{ }^{2}}+\varrho_{m}^{-1} \frac{\mathrm{d} A_{\mathrm{m}}}{\mathrm{d} \varrho_{\mathrm{m}}}-\left(1+4 \varrho_{\mathrm{m}}^{-2}\right) A_{\mathrm{m}} & =0, \\
\varrho_{\mathrm{m}} & =r L_{\mathrm{m}}^{-1} .
\end{aligned}
$$

Hence, $A_{\mathrm{m}}$ is a linear combination of the modified Bessel functions ${ }^{15}$ of index 2 ,

$A_{\mathrm{m}}(r)=\alpha_{\mathrm{m}} I_{2}\left(\frac{r}{L_{\mathrm{m}}}\right)+\gamma_{\mathrm{m}} K_{2}\left(\frac{r}{L_{\mathrm{m}}}\right)$.

By insertion of $(2.8)-(2.10)$ into the boundary condition (1.13),

$$
\mathbf{a}=C_{\mathrm{am}} p_{0}{ }^{-1} \overline{\boldsymbol{k}_{\mathrm{iso}}^{\mathrm{tan}} \boldsymbol{n}}-C_{\mathrm{a}} \boldsymbol{n} \cdot \underline{\mathbf{b}},
$$

the constants $\alpha_{\mathrm{m}}, \gamma_{\mathrm{m}}$ can be determined. The result is stated here only for small mean free paths $L_{\mathrm{m}}$, i. e. for $\left|L_{\mathrm{m}}\right| \ll R_{\mathrm{i}}$, where the asymptotic form ${ }^{15}$ of the modified Bessel functions can be used:

$r^{2} A_{\mathrm{m}}(r) \approx-\frac{\frac{1}{1+i m \varphi_{\mathrm{T}}}+\tilde{C}_{\mathrm{am}}}{1+E_{\mathrm{m}}}$.

$\cdot\left[1-\left(\frac{1-E_{\mathrm{m}}}{1+E_{\mathrm{m}}}\right)^{2} \exp \left(-2 \frac{R_{\mathrm{a}}-R_{\mathrm{i}}}{L_{m}}\right)\right]^{-1}$.

. $\left\{\left(\frac{r}{R_{\mathrm{a}}}\right)^{3 / 2} \exp \left(-\frac{R_{\mathrm{a}}-r}{L_{\mathrm{m}}}\right)\left[1-\frac{1-E_{\mathrm{m}}}{1+E_{\mathrm{m}}}\left(\frac{R_{\mathrm{a}}}{R_{\mathrm{i}}}\right)^{3 / 2}\right.\right.$.

$\left.\cdot \exp \left(-\frac{R_{\mathrm{i}}-R_{\mathrm{i}}}{L_{m}}\right)\right]+\left(\frac{r}{R_{\mathrm{i}}}\right)^{3 / 2} \exp \left(-\frac{r-R_{\mathrm{i}}}{L_{\mathrm{m}}}\right)$.

$\left.\cdot\left[1-\frac{1-E_{\mathrm{m}}}{1+E_{\mathrm{m}}}\left(\frac{R_{\mathrm{i}}}{R_{\mathrm{a}}}\right)^{3 / 2} \exp \left(-\frac{R_{\mathrm{a}}-R_{\mathrm{i}}}{L_{m}}\right)\right]\right\}$

with $\quad E_{\mathrm{m}} \equiv \tilde{C}_{\mathrm{a}}\left[\left(1+i m \varphi_{\mathrm{T}}\right) /\left(1+i m \varphi_{\mathrm{b}}\right)\right]^{1 / 2}$,

and $\quad C_{\mathrm{a}}=\sqrt{\frac{\omega_{\mathrm{b}}}{\omega_{\mathrm{T}}}} \tilde{C}_{\mathrm{a}}, \quad C_{\mathrm{am}}=\frac{\sqrt{2} \omega_{\eta \mathrm{T}}}{\omega_{\mathrm{T}}} \tilde{C}_{\mathrm{am}}$.

At the walls $\left(r=R_{\mathrm{i}}\right.$ and $\left.r=R_{\mathrm{a}}\right)$ both contributions in Eq. (2.9), $A_{\mathrm{m}}(r)$ and $r^{-2}\left(1+i m \varphi_{\mathrm{T}}\right)^{-1}$, to the $m$-component $\mathcal{P}^{(m)}: \mathbf{a}$ of the tensor polarization are of comparable size. But about some mean free paths $L_{\mathrm{m}} \mid$ away from the walls, $A_{\mathrm{m}}$ has decayed to zero. Within the gas mainly the term $r^{-2}\left(1+i m \varphi_{\mathrm{T}}\right)^{-1}$ survives since it is determined by collisions of the gas molecules with each other. In contradistinction, the contribution $A_{\mathrm{m}}(r)$ is determined by both, gasgas and gas-wall collisions. For high pressures and $r \neq R_{\mathrm{i}}, r \neq R_{\mathrm{a}}$ the function $A_{\mathrm{m}}(r)$ vanishes. 


\section{Flow Biref ringence}

(a) The general case

According to Eqs. (2.3), (2.8) and (2.9) the tensor polarization is given by

$$
\begin{aligned}
& \mathbf{a}=\frac{\sqrt{2} \omega_{\eta \mathrm{T}}}{\omega_{\eta} \omega_{\mathrm{T}}} \\
& \sum_{m=-2}^{+2}\left(\frac{1}{1+i m \varphi_{\mathrm{T}}}+r^{2} A_{\mathrm{m}}(r)\right) \boldsymbol{p}^{(m)}: \bar{\nabla}_{\text {iso }} .
\end{aligned}
$$

By the use of Eqs. (1.1) and (2.7) the anisotropic part of the dielectric tensor can then be expressed by the high pressure limit $\left(\bar{\nabla}_{\text {iso }}\right)_{\infty}$ of the velocity gradient,

$$
\bar{\varepsilon}=-2 \sum_{m=-2}^{+2} \beta^{(m)} \boldsymbol{p}^{(m)}:\left(\nabla \boldsymbol{v}_{\text {iso }}\right)_{\infty},
$$

with the coefficients

$$
\begin{array}{r}
\beta^{(m)}=\beta_{\infty}^{(m)} \frac{1+\left(1+i m \varphi_{\mathrm{T}}\right) r^{2} A_{\mathrm{m}}(r)}{1+2(l / d) C_{\mathrm{m}}}, \\
m=0, \pm 1, \pm 2 .
\end{array}
$$

In the high pressure limit $\left(p_{0} \rightarrow \infty\right.$ or $\left.l=0\right) \beta^{(m)}$ has the value ${ }^{5}$

$$
\beta_{\infty}^{(m)}=\frac{\beta}{1+i m \varphi_{\mathrm{T}}},
$$

and

$$
\begin{aligned}
& \beta=\beta_{\infty}^{(0)}=-\frac{\varepsilon^{\prime}}{2} \frac{\sqrt{2} \omega_{\eta \mathrm{T}}}{\omega_{\eta} \omega_{\mathrm{T}}} \\
& =\pi \frac{\alpha \|-\alpha \perp}{k T_{0}} \sqrt{\frac{2}{15}}\left(\left\langle J^{2}\left(J^{2}-\frac{3}{4}\right)^{-1}\right\rangle_{0}\right)^{1 / 2} \eta_{\mathrm{is} 0} \frac{\sqrt{2} \omega_{\eta \mathrm{T}}}{\omega_{\mathrm{T}}}
\end{aligned}
$$

is the pressure independent coefficient of flow birefringence ${ }^{2-5}$.

Inspection of Eq. (3.2) reveals that there are two contributions to the Knudsen corrections of the flow birefringence coefficients:

(i) One of them is the reduction factor $\left[1+2(l / d) C_{\mathrm{m}}\right]^{-1}$ of the velocity gradient according to mechanical slip.

(ii) The second contribution, $1+\left(1+i m \varphi_{\mathrm{T}}\right) r^{2} A_{\mathrm{m}}(r)$, is due to the influence of gas-wall collisions on the tensor polarization. It depends on the magnetic field and on the position $r$ of the light beam.

The parameters $C_{\mathrm{m}}, \tilde{C}_{\mathrm{a}}$ and $\tilde{C}_{\mathrm{am}}$ occurring in Eqs. (3.2) and (2.11) also describe Knudsen corrections for the Senftleben-Beenakker effect of vis- $\operatorname{cosity}^{6}$. They have already been determined ${ }^{6}$ for the gases $\mathrm{HD}, \mathrm{N}_{2}$ and $\mathrm{CO}$.

All experiments on flow birefringence in gases have been performed ${ }^{1}$ up to now without a magnetic field. Interest was focussed on the measurement of the high pressure value $\beta=\beta_{\infty}^{(0)}$ of the coefficient $\beta^{(\mathbf{0})}$, Knudsen corrections for $\beta$ have been studied in detail only for the gas $\mathrm{CO}_{2}$. From $\beta$, values of $\omega_{\eta \mathrm{T}} / \omega_{\mathrm{T}}$ have been derived for the gases $\mathrm{H}_{2}, \mathrm{HD}$, $\mathrm{N}_{2}, \mathrm{CO}$ and $\mathrm{CO}_{2}$. In particular, the sign of $\omega_{n \mathrm{~T}}$ was found to be positive. Furthermore, the absolute value $\left|\omega_{r \mathrm{~T}}\right| / \omega_{\mathrm{T}}$ was in reasonable agreement with that obtained from the Senftleben-Beenakker effect of viscosity ${ }^{10}$.

\section{(b) The special case $H=0$}

For zero magnetic field the principal axes of the dielectric tensor are ${ }^{3} \boldsymbol{e}$ and $\boldsymbol{e}_{ \pm}=1 / \sqrt{2}(\boldsymbol{u} \pm \boldsymbol{e} \times \boldsymbol{u})$. The difference in the index of refraction $\delta v=v_{+}-v$. for light travelling in $\boldsymbol{e}$-direction and polarized parallel to $\boldsymbol{e}_{+}$and $\boldsymbol{e}_{-}$, respectively, is given by ${ }^{3}$

$$
\delta v=\frac{1}{v} \beta^{(0)} \frac{2 \Omega_{\mathrm{i}}}{r^{2}\left(R_{\mathrm{i}}^{-2}-R_{\mathrm{a}}^{-2}\right)} .
$$

According to Eq. (3.2) the Knudsen corrections for $\delta v$ are then obtained as

$$
\delta v=(\delta v)_{\infty} \frac{1+r^{2} A_{0}(r)}{1+2(l / d) C_{\mathrm{m}}},
$$

here $(\delta v)_{\infty}$ denotes the high pressure limit of $\delta v$.

For $L_{0} / R_{\mathrm{i}} \ll 1$ Eq. $(2.11)$ supplies us with the result:

$$
\begin{aligned}
& \frac{\delta v}{(\delta v)_{\infty}}=\left\lfloor 1+2 \frac{l}{d} C_{\mathrm{m}}\right]^{-1}\left\{1-\frac{1+\tilde{C}_{\mathrm{am}}}{1+\tilde{C}_{\mathrm{a}}} .\right. \\
& \cdot\left[1-\left(\frac{1-\tilde{C}_{\mathrm{a}}}{1+\tilde{C}_{\mathrm{a}}}\right)^{2} \exp \left(-2 \frac{R_{\mathrm{a}}-R_{\mathrm{i}}}{L_{0}}\right)\right]^{-1} \cdot(3.7) \\
& \cdot \quad\left[( \frac { r } { R _ { \mathrm { a } } } ) ^ { 3 / 2 } \operatorname { e x p } ( - \frac { R _ { \mathrm { a } } - r } { L _ { 0 } } ) \left(1-\frac{1-\tilde{C}_{\mathrm{a}}}{1+\tilde{C}_{\mathrm{a}}}\left(\frac{R_{\mathrm{a}}}{R_{\mathrm{i}}}\right)^{3 / 2} .\right.\right. \\
& \left.\cdot \quad \exp \left(-\frac{R_{\mathrm{a}}-R_{\mathrm{i}}}{L_{0}}\right)\right)-\left(\frac{r}{R_{\mathrm{i}}}\right)^{3 / 2} \exp \left(-\frac{r-R_{\mathrm{i}}}{L_{9}}\right) \cdot \\
& \left.\left.\cdot\left(1-\frac{1-\tilde{C}_{\mathrm{a}}}{1+\tilde{C}_{\mathrm{a}}}\left(\frac{R_{\mathrm{i}}}{R_{\mathrm{a}}}\right)^{3 / 2} \exp \left(-\frac{R_{\mathrm{a}}-R_{\mathrm{i}}}{L_{0}}\right)\right)\right]\right\} .
\end{aligned}
$$

This complicated formula is considerably simplified if $\tilde{C}_{\mathrm{a}} \approx 1$ applies (e.g. $\tilde{C}_{\mathrm{a}}=1.0$ for ${ }^{6} \mathrm{~N}_{2}, \tilde{C}_{\mathrm{a}}=1.1$ for ${ }^{6} \mathrm{CO}$ ), or if the pressure is high enough so that 
$\exp \left(-\left(R_{\mathrm{a}}-R_{\mathrm{i}}\right) / L_{0}\right)$ can be neglected compared to 1 :

$$
\begin{aligned}
& \frac{\delta v}{(\delta v)_{\infty}}=\left[1+2 \frac{l}{d} C_{\mathrm{m}}\right]^{-1}\left\{1-\frac{1+\tilde{C}_{\mathrm{am}}}{1+\tilde{C}_{\mathrm{a}}}\left[\left(\frac{r}{R_{\mathrm{a}}}\right)^{3 / 2} .\right.\right. \\
& \left.\left.\cdot \exp \left(-\frac{R_{\mathrm{a}}-r}{L_{0}}\right)+\left(\frac{r}{R_{\mathrm{i}}}\right)^{3 / 2} \exp \left(-\frac{r-R_{\mathrm{i}}}{L_{0}}\right)\right]\right\} .
\end{aligned}
$$

In connection with the Senftleben-Beenakker effect of viscosity the Knudsen corrections could be obtained (in the special case $\omega_{\mathrm{T}}=\omega_{\mathrm{b}}$ ) simply by replacing all relaxation constants $\omega_{*}$ occurring in the hydrodynamic result for the constant pressure gradients by ${ }^{6}$

$$
\begin{gathered}
\left(\omega_{*}\right)_{\text {eff }}=\omega_{*}+\left(\omega_{*}\right)_{\text {Wall }}=\omega_{*}\left(1+l_{*} L^{-1}\right), \\
\varkappa=\eta, \mathrm{T}, \eta \mathrm{T} .
\end{gathered}
$$

The effective relaxation coefficient is the sum of a gas collision frequency $\omega_{\%}$ (which is proportional to pressure) and a pressure independent wall collision frequency $\left(\omega_{\varkappa}\right)_{\text {wall }}=\omega_{\varkappa} l_{\varkappa} L^{-1}$. Here, $l_{\varkappa}$ is a mean free path of a molecule and $L$ is a macroscopic length. In this way Hess ${ }^{9}$ gave a first estimate of the Knudsen corrections for flow and heat-flow birefringence. But from (3.7), (3.8) we see that this procedure is not applicable here, since it cannot account for the exponential decay of the function $A_{0}(r)$.

\section{(c) Pressure dependence of $\delta v$ for Baas' experimental arrangement}

For a calculation of the Knudsen corrections for $\delta v$ from Eqs. (3.7) or (3.8) the details of the apparatus (i.e. $R_{\mathrm{i}}, R_{\mathrm{a}}, r$ ), the mean free paths $l$ and $L_{0}$, and the surface parameters $C_{\mathrm{m}}, \tilde{C}_{\mathrm{a}}, \tilde{C}_{\mathrm{am}}$ have to be known. The arrangement used by Baas ${ }^{1}$ has the following dimensions:

$$
\begin{gathered}
R_{\mathrm{a}}=20.5 \mathrm{~mm}, \quad R_{\mathrm{i}}=16.67 \mathrm{~mm}, \\
r \equiv r_{1}=\frac{1}{2}\left(R_{\mathrm{a}}+R_{\mathrm{i}}\right)=18.58 \mathrm{~mm} .
\end{gathered}
$$

Hence we have $R_{\mathrm{a}}-R_{\mathrm{i}}=3.83 \mathrm{~mm}$ and $d=3.68 \mathrm{~mm}$.

The mean free paths $l$ and $L_{0}$ are calculated from the shear viscosity $\eta$ and from the relaxation constants $\omega_{\mathrm{T}}, \omega_{\mathrm{b}}$ which have been inferred ${ }^{7}$ from data ${ }^{10}$ on the Senftleben-Beenakker effect of viscosity and of thermal conductivity. For the gases $\mathrm{HD}, \mathrm{N}_{2}$ and $\mathrm{CO}$ the surface parameters $C_{\mathrm{m}}, \tilde{C}_{\mathrm{a}}$ and $\tilde{C}_{\text {am }}$ have already been determined ${ }^{6}$ from Knudsen corrections for the Senftleben-Beenakker effect of viscosity. In Table 1 values for some quantities relevant for flow birefringence are given.

In Fig. 1 the spatial variation of the tensor polarization

$\mathbf { a } = - \frac { \sqrt { 2 } \omega _ { \eta \mathrm { T } } } { \omega _ { \eta } \omega _ { \mathrm { T } } } \frac { 2 \Omega _ { \mathrm { i } } } { 1 - ( R _ { \mathrm { i } } / R _ { \mathrm { a } } ) ^ { 2 } } F ( r ) \longdiv { \boldsymbol { u } \boldsymbol { e } \times \boldsymbol { u } }$

and of the difference in the index of refraction

$$
\delta v=\frac{1}{v} \beta \frac{2 \Omega_{\mathrm{i}}}{1-\left(R_{\mathrm{i}} / R_{\mathrm{a}}\right)^{2}} F(r)
$$

is shown for the experimental set up used by Baas ${ }^{1}$. To this end the function

$$
F(r)=\frac{\left(R_{\mathrm{i}} / r\right)^{2}\left(1+r^{2} A_{0}(r)\right)}{1+2(l / d) C_{\mathrm{m}}}
$$

is plotted versus $r / R_{\mathrm{i}}$ for $\mathrm{HD}$ and for different pressures $p$. In the high pressure limit $(p \rightarrow \infty)$ we have

$$
\begin{aligned}
& F\left(R_{\mathrm{i}}\right) \equiv F_{\mathrm{i}}=1-\frac{1+\tilde{C}_{\mathrm{am}}}{1+\tilde{C}_{\mathrm{a}}}, \quad F\left(R_{\mathrm{a}}\right) \equiv F_{\mathrm{a}}= \\
& =\left(\frac{R_{\mathrm{i}}}{R_{\mathrm{a}}}\right)^{2} F_{\mathrm{i}}, \quad F(r) \rightarrow F_{\infty}(r)=\left(\frac{R_{\mathrm{i}}}{r}\right)^{2} \\
& \quad \text { for } \quad R_{\mathrm{i}}<r<R_{\mathrm{a}} .
\end{aligned}
$$

For finite pressures, i. e. $0<L_{0} \ll R_{\mathrm{a}}-R_{\mathrm{i}}$, the transition from the boundary values of $F$ to the value $F_{\infty} /\left(1+2(l / d) C_{\mathrm{m}}\right)$ within the gas occurs in a layer of thickness of some mean free paths $L_{0}$. At $p=0.0632$ Torr we have $L_{0}=R_{\mathrm{a}}-R_{\mathrm{i}}$, then the boundary layer covers the whole gap, and $F$ depends only weakly on $r$.

A light beam of diameter $1 \mathrm{~mm}$, centered at $r_{1}=\frac{1}{2}\left(R_{\mathrm{a}}+R_{\mathrm{i}}\right)$, passes the gap between $r_{0}=r_{1}+$ $0.5 \mathrm{~mm}$ and $r_{2}=r_{1}-0.5 \mathrm{~mm}$. If the center is at $r_{2}$ the beam passes between $r_{1}$ and $r_{3}=r_{1}-1.0 \mathrm{~mm}$.

The decrease of the tensor polarization due to wall effects leads to Knudsen corrections for $\delta v$. In Fig. 2 and in Fig. 3 the quantity $\delta v /(\delta v)_{\infty}$ is plotted versus pressure $p$ for $\mathrm{HD}$ and $\mathrm{N}_{2}$ respectively. The decrease of $\delta v$ with decreasing pressure is in qualitative agreement with Baas' ${ }^{1}$ observation for $\mathrm{CO}_{2}$. Since the values of $\omega_{\mathrm{b}}$ and of $\tilde{C}_{\mathrm{a}}, \tilde{C}_{\mathrm{am}}$ are not yet known for $\mathrm{CO}_{2}$ the corresponding curves cannot be drawn. The broken line in Fig. 2 and Fig. 3 represents the function $\left[1+2(l / d) C_{\mathrm{m}}\right]^{-1}$ which describes the reduction of the velocity gradient due to mechanical slip. The curves labelled by " $1,2,3$ " are calculated from Eq. (3.7) with $r=r_{1}, r_{2}, r_{3}$ re- 


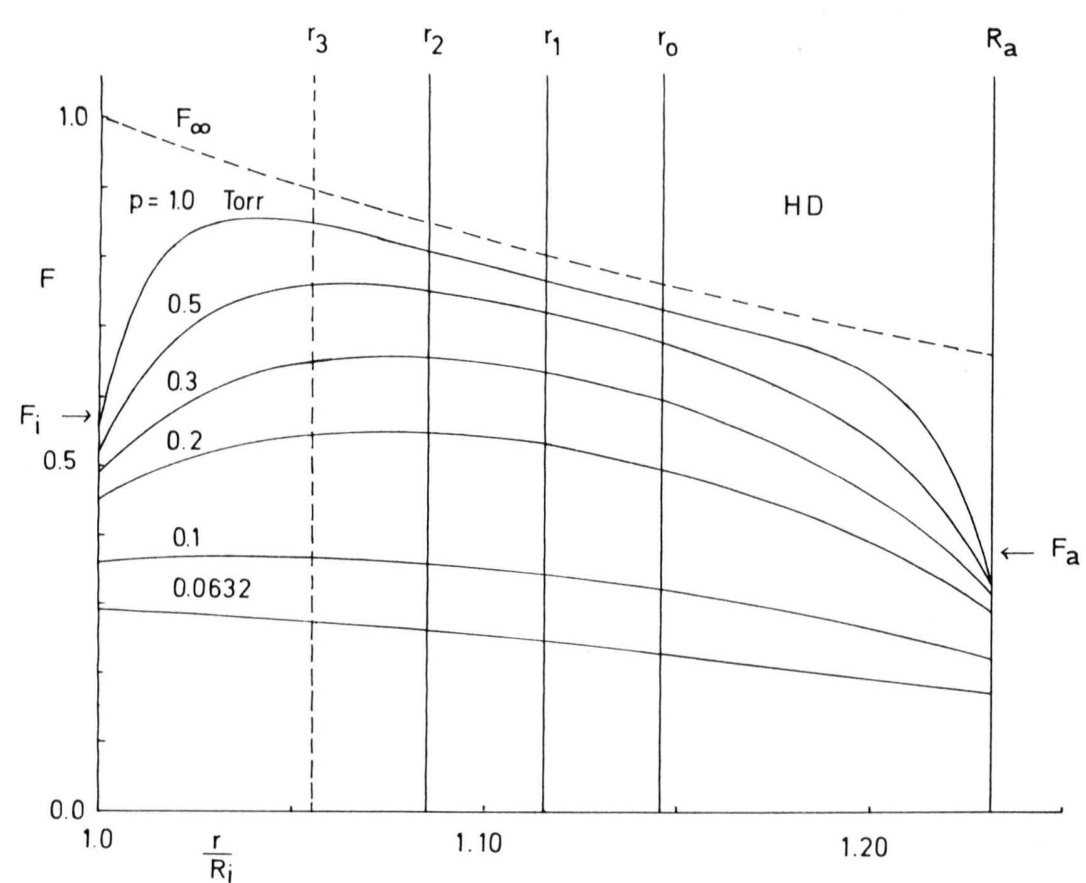

Fig. 1. Spatial variation of $\mathbf{a}$ and $\delta v$ for the gas HD and for $\boldsymbol{H}=\boldsymbol{O}$. The function $\boldsymbol{F}$ is calculated from Eqs. (3.11), (2.11) for Baas' apparatus ${ }^{1}$ with the parameters from Table 1 . The symbols $F_{\mathrm{i}}, F_{\mathrm{a}}, F_{\infty}$ refer to infinite pressure, see Equation (3.12). The light beam is centered at $r_{1}=\frac{1}{2}\left(R_{\mathrm{a}}+R_{\mathrm{i}}\right)$ and passes between $r_{2}=r_{1}-0.5 \mathrm{~mm}$ and $r_{0}=r_{1}+0.5 \mathrm{~mm}$. If the center is at $r_{2}$ the beam passes between $r_{1}$ and $r_{3}=r_{1}-1.0 \mathrm{~mm}$.

Table 1. Parameters for flow birefringence at $T_{0}=293 \mathrm{~K}$. The relaxation constants $\omega .$. have been inferred ${ }^{6,7}$ from viscosity and from data on the Senftleben-Beenakker effect of viscosity and of thermal conductivity. The sign of $\omega_{\eta} \mathrm{T}$ is positive according to Baas ${ }^{1}$. The surface parameters $C$. have been taken from Knudsen corrections for the Senftleben-Beenakker effect of viscosity ${ }^{6}$.

\begin{tabular}{|c|c|c|c|c|c|c|c|c|c|c|c|}
\hline & उ. & $\frac{\omega_{\mathrm{T}}}{\omega_{\eta}}$ & $\frac{\omega_{\mathrm{b}}}{\omega_{\eta}}$ & 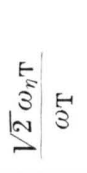 & 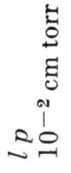 & 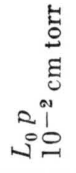 & $C_{a}$ & $C_{a m}$ & $C_{a}$ & $C_{a m}$ & $C_{m}$ \\
\hline HD & 0.96 & 0.12 & 0.73 & 0.18 & 0.91 & 2.43 & 1.00 & -0.07 & 0.4 & -0.4 & 1.0 \\
\hline $\mathrm{CO}$ & 0.59 & 0.88 & 1.33 & 0.09 & 0.49 & 0.35 & 1.38 & 0.0 & 1.1 & 0.0 & 1.2 \\
\hline $\mathrm{N}_{2}$ & 0.58 & 0.64 & 1.29 & 0.09 & 0.50 & 0.42 & 1.46 & 0.0 & 1.0 & 0.0 & 1.2 \\
\hline
\end{tabular}

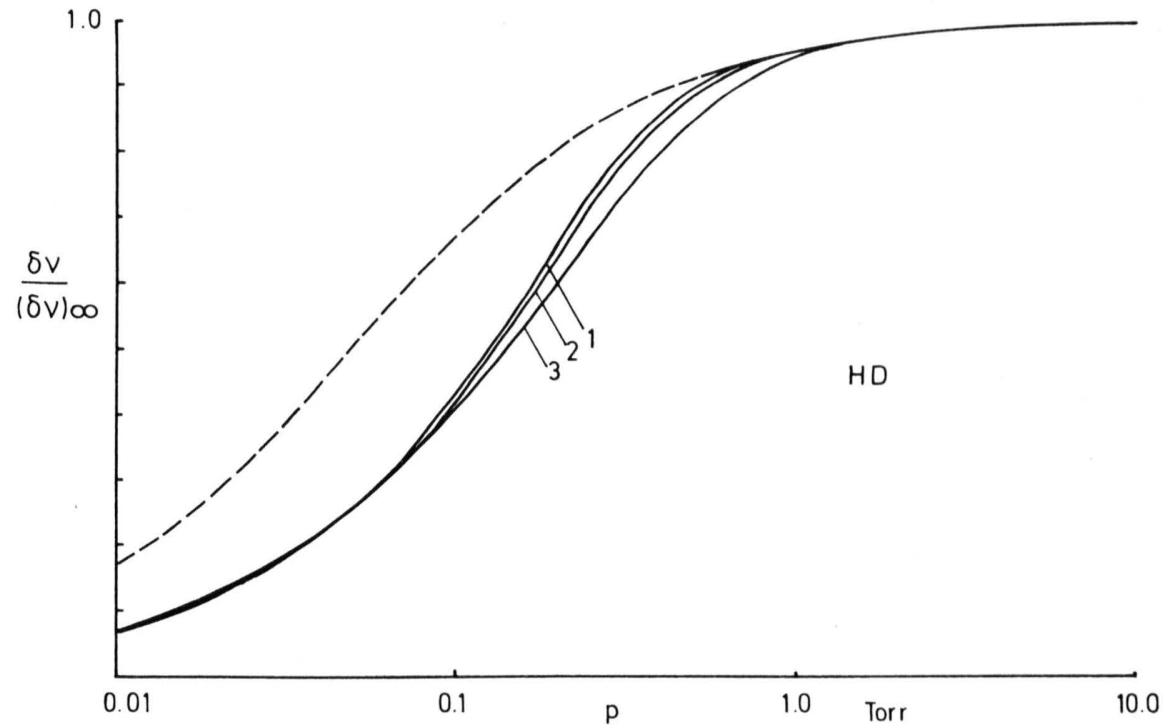

Fig. 2. Knudsen corrections for $\delta v$ as a function of pressure $p$. The quantity $\delta v /(\delta v)_{\infty}$ is calculated for HD from Eq. (3.7) for Baas' apparatus ${ }^{1}$ using the parameters from Table 1 . The curves labelled by " $1,2,3$ " refer to $r=r_{1}, r_{2}, r_{3}$ (see Figure 1). The broken line represents the function $\left[1+2(l / d) C_{\mathrm{m}}\right]^{-1}$.

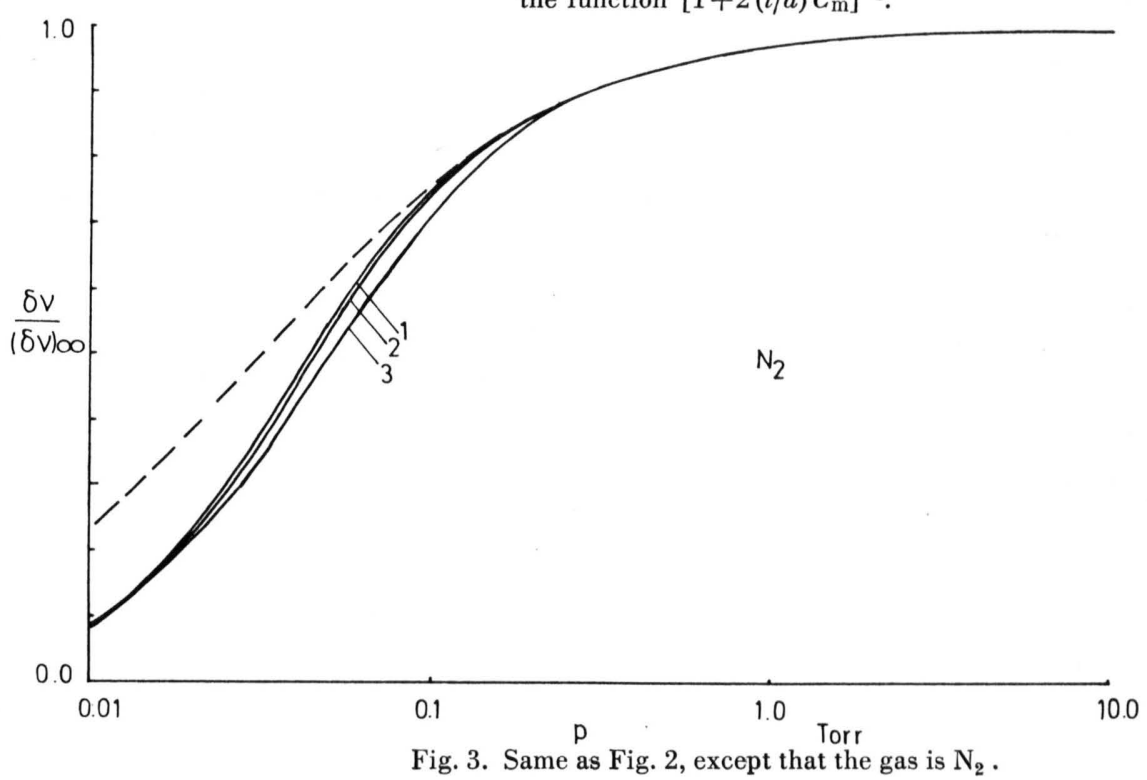


spectively. Experimental data should be found to lie between curves " 1 " and " 2 " (or between " 2 " and " 3 ") for a light beam of diameter $1 \mathrm{~mm}$ with the center at $r_{1}$ (or $r_{2}$ ). The distance between the broken line and curves " $1,2,3$ " is due to the influence of gas wall collisions on the tensor polarization. This influence shows up most clearly for the gas $\mathrm{HD}$ which has the largest mean free path $L_{0}$ (compare Fig. 2 for $\mathrm{HD}$ with Fig. 3 for $\mathrm{N}_{2}$ ). Furthermore, an analysis of the experimental data ${ }^{1}$ for $\mathrm{CO}_{2}$ reveals that the Knudsen corrections for $\delta y$ cannot be explained by the factor $\left[1+2(l / d) C_{\mathrm{m}}\right]^{-1}$ alone with a reasonable value for $C_{\mathrm{m}}$, i. e. $1 \leqq C_{\mathrm{m}}$ $\lesssim 1.5$.

Knudsen corrections for flow birefringence have been calculated from a solution of differential equations and boundary conditions for the velocity

1 F. Baas, Phys. Letters 36 A, 107 [1971]; Physica, to be published.

2 S. Hess, Phys. Letters 30 A, 239 [1969].

3 S. Hess, Springer Tracts in Mod. Phys. 54, 136 [1970].

4 S. Hess, in The Boltzmann Equation. Theory and Applications, eds. E. G. D. Cohen and W. Thirring, SpringerVerlag, Wien 1973.

5 A. G. St. Pierre. W. E. Köhler, and S. Hess, Z. Naturforsch. 27 a, 721 [1972].

${ }^{6}$ H. Vestner, Thesis, Transporterscheinungen und Randbedingungen in verdünnten mehratomigen Gasen im Magnetfeld. Erlangen 1973; Z. Naturforsch. 28 a, 869 [1973].

7 H. Vestner. Z. Naturforsch. 28 a, 1554 [1973].

8 W. E. Köhler and J. Halbritter, Z. Naturforsch., to be published.

9 S. Hess, in Proc. $7^{\text {th }}$ Int. Symp. Rarefied Gas Dynamics, Pisa 1970, ed. D. Dini, Academic Press, New York (in press) ; Z. Naturforsch. 28 a. 861 [1973]. and the tensor polarization. The influence of gaswall collissions on the tensor polarization can most clearly be seen for the gas HD. The same set of surface parameters describes Knudsen corrections for flow birefringence and for the Senftleben-Beenakker effect of viscosity. An experimental test of consistency for the theory could thus be derived from a measurement of both types of Knudsen corrections for the same gas.

\section{Acknowledgments}

I am indebted very much to Univ. Doz. Dr. S. Hess for discussions and for useful comments. Thanks are due to Dr. F. Baas for giving experimental details prior to publication.

${ }^{10}$ H. Senftleben, Phys. Z. 31, 822, 961 [1930]. - J. J. M. Beenakker, G. Scoles, H. F. P. Knaap, and R. M. Jonkman. Phys. Letters 2, 5 [1962]. - For a review and further reference see e. g. J. J. M. Beenakker and F. R. McCourt, Ann. Rev. Phys. Chem. 21, 47 [1970].

11 L. Waldmann, Z. Naturforsch. 12 a. 660 [1957]; 13 a, 609 [1958]. - R. F. Snider, J. Chem. Phys. 32, 1051 [1960].

12 S. Hess and L. Waldmann, Z. Naturforsch. 21 a, 1529 [1966]; 23 a, 1893 [1968]. - H. H. Raum and W. E. Köhler, Z. Naturforsch. 25 a. 1178 [1970].

13 L. Waldmann, Z. Naturforsch. 22 a, 1269 [1967].

14 S. Hess and L. Waldmann, Z. Naturforsch. 26 a. 1057 [1971].

15 M. Abramowitz and I. A. Stegun, Handbook of Mathemati cal Functions, Dover Publications, New York 1965. 